\title{
AOMAR ANANE \\ OMAR CHAKRONE \\ Sur un théorème de point critique et application à un problème de non-résonance entre deux valeurs propres du $p$-laplacien
}

Annales de la faculté des sciences de Toulouse $\sigma^{e}$ série, tome $9, \mathrm{n}^{\circ} 1$ (2000), p. 5-30

$<$ http://www.numdam.org/item?id=AFST_2000_6_9_1_5_0>

(C) Université Paul Sabatier, 2000, tous droits réservés.

L'accès aux archives de la revue «Annales de la faculté des sciences de Toulouse » (http://picard.ups-tlse.fr/ annales/) implique l'accord avec les conditions générales d'utilisation (http://www.numdam.org/conditions). Toute utilisation commerciale ou impression systématique est constitutive d'une infraction pénale. Toute copie ou impression de ce fichier doit contenir la présente mention de copyright.

\section{NumDam}

Article numérisé dans le cadre du programme

Numérisation de documents anciens mathématiques

http://www.numdam.org/ 


\title{
Sur un théorème de point critique et application à un problème de non-résonance entre deux valeurs propres du p-laplacien $\left.{ }^{*}\right)$
}

\author{
Aomar Anane ${ }^{(1)}$ Et Omar Chakrone ${ }^{(1)}$
}

\begin{abstract}
Résumé. - Nous établissons un théorème abstrait de point critique qui constitue une généralisation des théorèmes de liaisons classiques. Nous établissons également quelques caractérisations variationnelles du spectre du p-laplacien. Comme applications nous démontrons l'existence de solution du problème $(\mathcal{P})$ avec des conditions sur le comportement des rapports $\frac{f(x, s)}{|s|^{p-2} s}$ et $\frac{p F(x, s)}{|s|^{p}}$ entre deux valeurs propres consécutives du p-laplacien.
\end{abstract}

Abstract. - We establish an abstract theorem of critical point which constitute a generalization of classical links theorems. We also establish some variational characterizations of the p-laplacian spectrum. As application we prove the existence of solution of the problem $(\mathcal{P})$ :

$$
-\Delta_{p} u=f(x, u) \quad \text { dans } \Omega, \quad u=0 \quad \text { sur } \partial \Omega,
$$

with conditions on the behaviour of the ratios : $\frac{f(x, s)}{|s|^{p-2} s}$ and $\frac{p F(x, s)}{|s|^{p}}$ between two consecutive eigenvalues values of the p-laplacian where $F$ is a potential of $f$.

(*) Reçu le 9 mars 1998, accepté le 28 février 2000

(1) Université Mohamed I, Faculté des Sciences, Département de Mathématiques et Informatique, Oujda, Maroc.

e-mail: anane@sciences.univ-oujda.ac.ma

chakrone@sciences.univ-oujda.ac.ma 


\section{Introduction}

Considérons le problème de Dirichlet

$$
\left\{\begin{aligned}
-\Delta_{p} u & =f(x, u)+h & \text { dans } & \Omega, \\
u & =0 & \text { sur } & \partial \Omega
\end{aligned}\right.
$$

où $\Omega$ est un domaine borné de $\mathbb{R}^{N}, 1<p<+\infty, \Delta_{p} u=\operatorname{div}\left(|\nabla u|^{p-2} \nabla u\right)$ représente le p-laplacien, $h \in W^{-1, p^{\prime}}(\Omega)$ et $f: \Omega \times \mathbb{R} \rightarrow \mathbb{R}$ est une fonction de Carathéodory.

On s'intéresse à l'étude de l'existence de solutions du problème (1.1) sous les hypothèses

$$
\begin{aligned}
& \left(f_{\alpha, \beta}\right) \quad \alpha \leqslant \liminf _{s \rightarrow \pm \infty} \frac{f(x, s)}{|s|^{p-2} s} \text { et } \limsup _{s \rightarrow \pm \infty} \frac{f(x, s)}{|s|^{p-2} s} \leqslant \beta, \\
& \left(\mathbf{F}_{\alpha, \beta}\right) \quad \alpha \leqslant l \equiv l(x):=\liminf _{s \rightarrow \pm \infty} \frac{p F(x, s)}{|s|^{p}}, \limsup _{s \rightarrow \pm \infty} \frac{p F(x, s)}{|s|^{p}}:=k(x) \leqslant \beta \\
& \text { p.p. } x \in \Omega \text { et }
\end{aligned}
$$$$
\forall \varepsilon>0, \exists d_{\varepsilon} \in L^{1}(\Omega) \text { tel que p.p. } x \in \Omega, \forall s \in \mathbb{R} \text {, on a }
$$$$
-d_{\varepsilon}(x)+(l(x)-\varepsilon) \frac{|s|^{p}}{p} \leqslant F(x, s) \leqslant(k(x)+\varepsilon) \frac{|s|^{p}}{p}+d_{\varepsilon}(x)
$$

où $\alpha=\lambda_{n}$ et $\beta=\lambda_{n+1}$ sont deux valeurs propres consécutives du p-laplacien et $F: \Omega \times \mathbb{R} \rightarrow \mathbb{R}$ est une fonction définie par $F(x, s)=\int_{0}^{s} f(x, t) d t$.

La notation $\leqslant \not \equiv$ signifie qu'on a une inégalité large p.p. sur $\Omega$ et stricte sur un sous ensemble de $\Omega$ de mesure non nulle.

On considère des conditions qui assurent l'existence d'une solution $u \in$ $W_{0}^{1, p}(\Omega)$, pour tout $h \in W^{-1, p^{\prime}}(\Omega)$ (non-résonance).

Cette étude est consacrée initialement à l'établissement d'un résultat de point critique, de type minimax pour la fonctionnelle énergie $\Phi_{p}$ associée au problème (1.1) définie par

$$
\Phi_{p}(u)=\frac{1}{p} \int_{\Omega}|\nabla u|^{p}-\int_{\Omega} F(x, u)-\langle h, u\rangle
$$

où $\langle h, u\rangle$ désigne le produit de dualité d'un élément $h \in W^{-1, p^{\prime}}(\Omega)$ par $u \in W_{0}^{1, p}(\Omega)$. (Nous ferons des hypothèses sur $f$, pour que $\Phi_{p}$ soit bien définie et de classe $C^{1}$ sur $\left.W_{0}^{1, p}(\Omega)\right)$. 
Dans le cas linéaire $p=2$ (cas du laplacien), les premiers travaux dans cette direction utilisant une approche variationnelle sont dûs à Dolph [Do 1949]. Ensuite plusieurs études ont été faites sur ce type de problème, citons par exemple $[\mathrm{Ah}, \mathrm{La}, \mathrm{Pa}],[\mathrm{Br}, \mathrm{Ni}],[\mathrm{Th}],[\mathrm{Ca}],[\mathrm{Mo}],[\mathrm{Go}, \mathrm{Mo}]$ et $[\mathrm{Co}, \mathrm{Ol}]$.

Les méthodes utilisées dans ces travaux font intervenir essentiellement le caractère linéaire du laplacien et la décomposition de l'espace de Hilbert $W_{0}^{1,2}(\Omega)$ en somme directe d'un sous espace vectoriel $V$ engendré par les $n$ premières fonctions propres de $-\Delta$ et de son orthogonale $W\left(W_{0}^{1,2}(\Omega)=\right.$ $V \oplus W)$. La caractérisation variationnelle de la valeur propre $\lambda_{n+1}$ :

$$
\frac{1}{\lambda_{n+1}}=\sup \left\{\frac{\int_{\Omega}|u|^{2}}{\int_{\Omega}|\nabla u|^{2}} \quad u \in W \backslash\{0\}\right\},
$$

permet d'établir la coercivité et l'anticoercivité de la fonctionnelle $\Phi_{2}$ sur $W$ et $V$ respectivement, ce qui conduit à l'existence d'une valeur critique de type minimax de $\Phi_{2}$ en utilisant le théorème de point selle de Rabinowitz[Ra] ou bien le théorème de liaison[Z].

Dans le cas où l'opérateur est non linéaire $(p \neq 2)$, les résultats connus de natures variationnelles sont démontrés lorsque les quotients $\frac{f(x, s)}{|s|^{p-2} s}$ et $\frac{p F(x, s)}{|s|^{p}}$ se situent à gauche de la première valeur propre $\lambda_{1}$ du p-laplacien $\left(-\Delta_{p}\right)$ (voir $\left.[\mathrm{An}, \mathrm{Go}]\right)$. Ils utilisent la caractérisation variationnelle de $\lambda_{1}$ :

$$
\frac{1}{\lambda_{1}}=\sup \left\{\frac{\int_{\Omega}|u|^{p}}{\int_{\Omega}|\nabla u|^{p}} \quad u \in W_{0}^{1, p}(\Omega) \backslash\{0\}\right\}
$$

Rappelons que les nombres $\lambda_{n}(\gamma)$ définis par :

$$
\frac{1}{\lambda_{n}(\gamma)}=\sup _{K \in A_{n}(\gamma)} \min _{u \in K} \int_{\Omega}|u|^{p},
$$

appartiennent au spectre $\sigma\left(-\Delta_{p}\right)$ du p-laplacien (voir [An2]), où $\gamma(F)$ est le genre d'une partie $F \subset W_{0}^{1, p}(\Omega) \backslash\{0\}$ fermée et symétrique, $A_{n}(\gamma)=\{K \subset$ $S ; K$ compact symétrique et $\gamma(k) \geqslant n\}$ et $S=\left\{u \in W_{0}^{1, p}(\Omega) ;\|u\|_{1, p}=\right.$ 1\}. Anane[An1] a montré que $\lambda_{1}$ est simple et isolée, ensuite Anane et Tsouli[An,Ts1] ont montré que $\lambda_{2}$ est bien la deuxième valeur propre, c'està-dire $\lambda_{1}<\lambda_{2}$ et $] \lambda_{1}, \lambda_{2}\left[\cap \sigma\left(-\Delta_{p}\right)=\emptyset\right.$.

D'autre travaux ont été effectués dans cette direction utilisant une méthode topologique (degré topologique). Citons par exemple :

$$
\text { - }[\mathrm{Bo}, \mathrm{Dr}, \mathrm{Da}, \mathrm{Ku}] \text { dans le cas } N=1,1<p<+\infty \text { et }\left(f_{\mu_{n}+\delta, \mu_{n+1}-\delta}\right) \text { pour }
$$


un certain $\delta>0$ où $\mu_{n}$, et $\mu_{n+1}$ sont deux valeurs propres consécutives du p-laplacien.

- [An,Ts2] dans le cas $N \geqslant 11<p<+\infty$ et

$$
\lambda_{1} \leqslant \not \equiv \liminf _{s \rightarrow \pm \infty} \frac{f(x, s)}{|s|^{p-2} s} \text { et } \limsup _{s \rightarrow \pm \infty} \frac{f(x, s)}{|s|^{p-2} s}<\lambda_{2}
$$

où $\lambda_{1}$ et $\lambda_{2}$ sont la première et la deuxième valeur propre du p-laplacien.

Notons que les conditions que nous considérons sont plus faibles que celles considérer par les auteurs précédents. Cette amélioration est due à la méthode variationnelle utilisée lors de la résolution du problème. (C'està-dire : L'hypothèse $\left(F_{\alpha, \beta}\right)$ est satisfaite dès que la fonction $f$ vérifie la condition $\left(f_{\alpha, \beta}\right)$ en remplaçant $\leqslant$ par $\leqslant \not \equiv$. Mais on démontre facilement que l'inverse est généralement faux).

Il est clair que les méthodes utilisées dans le cas linéaire $p=2$ ne s'adaptent pas à notre situation, d'où la nécessité de l'établissement d'un théorème de point critique adéquat.

Ce travail est organisé en trois parties :

Dans la première partie, nous démontrons un résultat abstrait de point critique d'une fonctionnelle $\Phi$ définie sur un espace de Banach $X$ (théorème 1 , section 2). Les valeurs critiques sont données par le principe de minimax :

$$
c=\inf _{h \in \Gamma} \max _{u \in h\left(\overline{\left.c o\left(K_{0}\right)\right)}\right.} \Phi(u)
$$

où $c o\left(K_{0}\right)$ est l'enveloppe convexe d'un compact $K_{0}$ de $X$, et $\Gamma=\{h \in$ $\left.C\left(\overline{c o\left(K_{0}\right)}, X \backslash\{0\}\right) ; h(x)=x \quad \forall x \in K_{0}\right\}$, avec une condition de liaison de la forme $h\left(\overline{c o\left(K_{0}\right)}\right) \cap A \neq \emptyset$ pour tout $h \in \Gamma$ où $A$ est une partie de $X$ non vide vérifiant : $\max _{K_{0}} \Phi<\inf _{A} \Phi$, et la condition usuelle de PalaisSmale $(P S)$.

Un corollaire de ce théorème est établi dans la section 3 , il fait intervenir une nouvelle fonction genre $\theta$ "plus fine que $\gamma$ ». La condition de liaison précédente sera satisfaite lorsque $K \cap A \neq \emptyset$ pour tout $K$ vérifiant $\theta(K) \geqslant$ $\theta\left(K_{0}\right)+1$ (dans ce cas $A$ et $K_{0}$ sont symétriques). Ce corollaire constitue une généralisation des théorèmes de liaison[Z] et de point selle de Rabinowitz[Ra] (Remarque 1).

Dans la 2ème partie (section 4), nous établissons quelques caractérisations variationnelles du spectre du p-laplacien. Nous montrons que : 
Sur un théorème de point critique et application à un problème de non-résonance

$-\left(\lambda_{n}(\theta)\right) \subset \sigma\left(-\Delta_{p}\right)$ où $\theta$ est la fonction introduite dans la section 3 et $\lambda_{n}(\theta)$ est donnée par (1.4) en remplaçant $\gamma \operatorname{par} \theta$.

- Si $N \geqslant 1$ et $1<p<+\infty$, alors $\lambda_{i}=\lambda_{i}(\theta)=\lambda_{i}(\gamma)$ pour $i=1,2$.

- Si $p=2$ et $N \geqslant 1$, alors $\lambda_{n}=\lambda_{n}(\theta)=\lambda_{n}(\gamma)$ pour tout $n \geqslant 1$ où la suite $\left(\lambda_{n}\right)$ constitue le spectre du laplacien $-\Delta$.

- Si $N=1$ et $1<p<+\infty$, alors $\mu_{n}=\lambda_{n}(\theta)=\lambda_{n}(\gamma)$ pour tout $n \geqslant 1$ où $\left(\mu_{n}\right)$ est la suite introduite dans [Ot1] où [Ot2] définie par : $\mu_{n}=n^{p}(p-1)\left\{\frac{1}{\pi} \int_{0}^{1} \frac{d t}{\left(1-t^{p}\right)^{\frac{1}{p}}}\right\}^{p}$ qui constitue le spectre du p-laplacien.

Enfin, la $3^{\mathrm{e}}$ partie (les sections 5 et 6 ) est consacrée à l'étude de l'existence de solution pour le problème (1.1). Deux types de conditions interviennent : La condition usuelle de (PS), que nous étudions dans la section 5, qui n'est pas l'objet principale de notre travail, et les conditions géométriques. Pour traiter ces dernières, nous appliquons d'une part le corollaire du théorème (lère partie), et d'autre part la caractérisation variationnelle des valeurs propres $\lambda_{1}, \lambda_{2}$ et $\mu_{n}$ pour tout $n \geqslant 1$ (2ème partie).

Nous établissons dans la section 6 des résultats d'existences de solutions du problème (1.1) par un procédé de minimax appliqué à la fonctionnnelle $\Phi_{p}$ dans les situations suivantes (théorème 6) :

$-N=1,1<p<+\infty,\left(f_{\mu_{n}, \mu_{n+1}}\right)$ et $\left(F_{\mu_{n}, \mu_{n+1}}\right)$. Ce qui constitu une amélioration du résultat établi dans $[\mathrm{Bo}, \mathrm{Dr}, \mathrm{Da}, \mathrm{Ku}]$.

$-N>1,1<p<+\infty,\left(f_{\lambda_{1}, \lambda_{2}}\right)$ et $\left(F_{\lambda_{1}, \lambda_{2}}\right)$ avec $k(x)<\lambda_{2}$ p.p. $x \in \Omega$. Ces conditions sont meilleures que celles considérées dans [An,Ts2].

$-N>1,1<p<+\infty$ et $f(x, s)=\lambda m(x)|s|^{p-2} s$ où $m$ une fonction poids bornée qui peut changer de signe et tel que $\lambda \notin \sigma\left(-\Delta_{p}, 1\right)$ (Alternative de Fredholme non linéaire).

- $N>1, p=2,\left(f_{\lambda_{n}, \lambda_{n+1}}\right)$ et $\left(F_{\lambda_{n}, \lambda_{n+1}}\right)$ où $\lambda_{n}$ et $\lambda_{n+1}$ sont deux valeurs propres consécutives du laplacien. On retrouve ainsi le résultat de $[\mathrm{Co}, \mathrm{Ol}]$ par une méthode non-linéaire.

\section{Théorème abstrait}

Désignons par $X$ un espace de Banach réel de dimension infinie et $c o(K)$ l'enveloppe convexe d'une partie $K$ de $X$. Rappelons qu'une fonctionnelle $\Phi: X \rightarrow \mathbb{R}$ de classe $C^{1}$ satisfait la condition de (PS) sur $X$ si pour toute suite $\left(u_{n}\right)$ de $X$ telle que la suite $\left(\Phi\left(u_{n}\right)\right)$ est bornée, et $\Phi^{\prime}\left(u_{n}\right) \rightarrow 0$ dans $X^{\prime}$, alors $\left(u_{n}\right)$ possède une sous suite convergente dans $X$. 
THÉORÈME 1. - Soient $\Phi \in C^{1}(X, \mathbb{R})$ une fonctionnelle satisfaisant la condition (PS) sur $X, K_{0}$ un compact non vide tel que $0 \notin K_{0}$ et $A \subset X$ un ensemble non vide. Si les conditions suivantes sont vérifiées

$\left(\mathbf{P}_{1}\right) h(\bar{D}) \cap A \neq \emptyset \quad \forall h \in \Gamma$ où $\Gamma=\left\{h \in C(\bar{D}, X \backslash\{0\}) ; h_{/ K_{0}}=i d\right\}$ et $D=c o\left(K_{0}\right)$.

$\left(\mathbf{P}_{2}\right) \quad \alpha:=\max _{K_{0}} \Phi<\inf _{A} \Phi:=\beta$.

Alors la valeur

$$
c:=\inf _{h \in \Gamma} \max _{u \in h(\bar{D})} \Phi(u)
$$

est une valeur critique de la fonctionnelle $\Phi$. De plus, on a

$$
c \geqslant \beta \text {. }
$$

Pour justifier la bonne définition de la valeur $c$, on a

Lemme $1 .-\Gamma \neq \emptyset$ où $\Gamma$ est défini dans le théorème 1.

Preuve du lemme 1. - Si $0 \notin \bar{D}$, alors $i d \in \Gamma$, par suite le lemme est démontré. Supposons maintenant que $0 \in \bar{D}$.

1ère étape. Montrons qu'il existe $v \in X \backslash\{0\}$ tel que $D_{v} \cap K_{0}=\emptyset$ où $D_{v}=\{t v, t \geqslant 0\}$.

En effet, soit $r>0$ tel que $\bar{B}(0, r) \cap K_{0}=\emptyset$, comme $\operatorname{dim} X=+\infty$ et $\bar{D}$ est compact, il est clair que $\partial B(0, r) \backslash \bar{D} \neq \emptyset$. Soit $v \in \partial B(0, r) \backslash \bar{D}$, nous avons $D_{v} \cap K_{0}=\emptyset$. En effet, si $D_{v} \cap K_{0} \neq \emptyset$, alors il existe $t>0$ tel que $t v \in K_{0}$, puisque $\bar{B}(0, r) \cap K_{0}=\emptyset$, alors $\|t v\|>r$, or $\|v\|=r$, de sorte que $t>1$ et que $v \in[0, t v]$, d'où $v \in \bar{D}$, ce qui est absurde.

2ème étape. Montrons que $\Gamma \neq \emptyset$.

Soit $b>0$ tel que $K_{0} \subset \bar{B}(0, b)$. Posons $\varepsilon=d\left(D_{v}, K_{0}\right)=\min _{u \in K_{0}, t \geqslant 0}\|t v-u\|$, on a $0<\varepsilon<+\infty$. Posons $\alpha=\frac{2 b}{\varepsilon\|v\|}$ et définissons $h: \bar{D} \rightarrow X: h(x)=$ $x-\alpha d\left(x, K_{0}\right) v$. Il est clair que $h$ est continue sur $\bar{D}$ et que $h(x)=x$ pour tout $x \in K_{0}$. Pour terminer montrons que $h(x) \neq 0 \forall x \in \bar{D}$. Supposons, par l'absurde, qu'il existe $x \in \bar{D}$ tel que $h(x)=0$, donc $x=\alpha d\left(x, K_{0}\right) v$, de sorte que $x \in D_{v}$. D'autre part, on a

$$
\begin{aligned}
\|x\| & =\alpha d\left(x, K_{0}\right)\|v\| \\
& =2 b \frac{d\left(x, K_{0}\right)}{\varepsilon} \\
& \geqslant 2 b,
\end{aligned}
$$


Sur un théorème de point critique et application à un problème de non-résonance

donc $x \notin \bar{B}(0, b)$, par suite $x \notin \bar{D}$, ce qui est absurde. D'où la preuve du lemme 1 .

Preuve du théorème 1. - On peut supposer, sans perte de généralité, que $\Phi(0)=0$. En effet, écrivons $\Phi(u)=(\Phi(u)-\Phi(0))+\Phi(0)$, alors $c$ est une valeur critique de $\Phi$ si et seulement si $c-\Phi(0)$ est une valeur critique de $\Phi-\Phi(0)$.

Soit $h \in \Gamma$, d'après $\left(P_{1}\right)$ il existe $u_{0} \in h(\bar{D}) \cap A$, donc la définition de la valeur $\beta$ implique $\beta \leqslant \Phi\left(u_{0}\right) \leqslant \max _{u \in h(\bar{D})} \Phi(u)$, comme $h$ est arbitraire, on en conclut que $c \in \mathbb{R}$ et $\beta \leqslant c$.

Pour montrer que $c$ est une valeur critique, supposons, par l'absurde, que $K_{c}=\left\{u \in X ; \Phi(u)=c\right.$ et $\left.\Phi^{\prime}(u)=0\right\}=\emptyset$ et choisissons $\bar{\varepsilon}>0$ tel que

$$
\begin{cases}\bar{\varepsilon}<\min \left\{\frac{1}{2}(\beta-\alpha), c\right\} & \text { si } \quad c>0 \\ \bar{\varepsilon} \leqslant \frac{1}{2}(\beta-\alpha) & \text { si } \quad c \leqslant 0\end{cases}
$$

Puisque $\Phi$ satisfait la condition de (PS) sur $X$, le théorème de déformation (voir [Ra]) montre qu'il existe $\varepsilon \in] 0, \bar{\varepsilon}[$ et $\eta \in C([0,1] \times X, X)$ vérifiant :

$\left(D_{1}\right)$ Si $\Phi(u) \notin[c-\bar{\varepsilon}, c+\bar{\varepsilon}]$, alors $\eta(t, u)=u$ pour tout $t \in[0,1]$.

$\left(D_{2}\right) \eta(t, u)$ est un homéomorphisme de $X$ sur $X$ pour tout $t \in[0,1]$.

$\left(\mathbf{D}_{3}\right) \eta\left(1, \Phi^{c+\varepsilon}\right) \subset \Phi^{c-\varepsilon}$ où $\Phi^{s}=\{u \in X$ tel que $\Phi(u) \leqslant s\}$.

Par définition de la valeur $c$, il existe $h \in \Gamma$ tel que $\max _{u \in h(\bar{D})} \Phi(u) \leqslant$ $c+\varepsilon$, donc $h(\bar{D}) \subset \Phi^{c+\varepsilon}$, par $\left(D_{3}\right)$, on a $\eta(1, h(\bar{D})) \subset \Phi^{c-\varepsilon}$, il vient que

$$
\max _{u \in \tilde{h}(\bar{D})} \Phi(u) \leqslant c-\varepsilon
$$

où $\tilde{h}: \bar{D} \rightarrow X: \tilde{h}(u)=\eta(1, h(u))$ pour tout $u \in \bar{D}$. Si on montre que $\tilde{h} \in \Gamma$, alors on aura $c \leqslant \max _{u \in \tilde{h}(\bar{D})} \Phi(u) \leqslant c-\varepsilon$, ce qui est absurde. Pour montrer ceci, il suffit de vérifier que $\tilde{h}(u) \neq 0$ pour tout $u \in \bar{D}$ et que $\tilde{h}(u)=u$ pour tout $u \in K_{0}$. En effet, si $c \leqslant 0$, alors par (2.1), on a $\Phi(\tilde{h}(v)) \leqslant c-\varepsilon<0$ pour tout $u \in \bar{D}$, par suite $\tilde{h}(v) \neq 0$ pour tout $u \in \bar{D}(\operatorname{car} \Phi(0)=0)$. Si $c>0$, alors $\Phi(0)=0<c-\bar{\varepsilon}$, et par $\left(D_{1}\right)$, on a $\eta(1,0)=0$, or $h(u) \neq 0$ pour tout $u \in \bar{D}$, donc $\operatorname{par}\left(D_{2}\right) \tilde{h}(u)=\eta(1, h(u)) \neq 0$ pour tout $u \in \bar{D}$. D'autre part, soit $u \in K_{0}$, comme $\beta \leqslant c$, et d'après l'hypothèse $\left(P_{2}\right)$ et le choix de $\bar{\varepsilon}$, on a

$$
\Phi(u) \leqslant \alpha<\alpha+\bar{\varepsilon} \leqslant \beta-\frac{\beta-\alpha}{2} \leqslant c-\bar{\varepsilon},
$$

de sorte que $\Phi(u) \notin[c-\bar{\varepsilon}, c+\bar{\varepsilon}]$. Enfin d'après $\left(D_{1}\right)$, on obtient $\eta(1, u)=u$, il vient que $\tilde{h}(u)=\eta(1, h(u))=\eta(1, u)=u$, il en résulte que $\tilde{h} \in \Gamma$, d'où le théorème 1. 


\section{Corollaire du théorème 1}

Nous allons établir dans cette section un corollaire du théorème 1 qui donne une situation particulière et interéssante pour les applications.

Notons par $\operatorname{Sym}(X)$ l'ensemble des parties $F$ de $X$ fermées symétriques telles que $0 \notin F$ et rappelons la définition du genre $\gamma(F)$ d'un élément $F$ de $\operatorname{Sym}(X)$ (dont on trouve les propriétés dans [Ze] par exemple).

Définition 1. - La fonction genre $\gamma: \operatorname{Sym}(X) \rightarrow \mathbb{N} \cup\{+\infty\}$ est définie par

1) $\gamma(\emptyset)=0$,

2) si $F \neq \emptyset$, alors $\gamma(F)=k$ est le plus petit entier tel qu'il existe une application

$\phi: F \rightarrow \mathbb{R}^{k} \backslash\{0\}$ impaire et continue,

3) $\gamma(F)=+\infty$ si une telle application n'existe pas.

Remarque 1. - Dans la définition 1 l'ensemble $\mathbb{R}^{k} \backslash\{0\}$ peut être remplacé par

$S^{k-1}=\left\{x \in \mathbb{R}^{k} ;\|x\|_{\mathbb{R}^{k}}=1\right\}$.

En effet, si $\phi: F \rightarrow \mathbb{R}^{k} \backslash\{0\}$ est une application impaire et continue, alors l'application $g=h \circ \phi: F \rightarrow S^{k-1}$ est impaire continue, où $h: \mathbb{R}^{k} \backslash\{0\} \rightarrow$ $S^{k-1}: h(x)=\frac{x}{\|x\|_{\mathbb{R}^{k}}}$.

Ceci nous conduit à introduire une nouvelle fonction genre $\theta: \operatorname{Sym}(X) \rightarrow$ $\mathbb{N} \cup\{+\infty\}:$

Définition 2. - La fonction $\theta: \operatorname{Sym}(X) \rightarrow \mathbb{N} \cup\{+\infty\}$ est définie par

1) $\theta(\emptyset)=0$,

2) Si $F \neq \emptyset$, alors $\theta(F)=\sup \left\{k \in \mathbb{N}\right.$; il existe $f \in C\left(S^{k-1}, F\right)$ impaire $\}$.

Remarquons que si $F \neq \emptyset$, alors $1 \in\left\{k \in \mathbb{N}\right.$; il existe $f \in C\left(S^{k-1}, F\right)$ impaire $\}$, la définition 2 a donc un sens.

Nous ne savons pas si la fonction $\theta$ coïncide avec le genre usuel $\gamma$, néanmoins, elle vérifie les propriétés suivantes qui sont déjà satisfaites par $\gamma$.

Proposition 1. - Soit $K, K_{1}, K_{2} \in \operatorname{Sym}(X)$, on a

1) $\theta(K) \leqslant \gamma(K)$. 
Sur un théorème de point critique et application à un problème de non-résonance

2) $\theta\left(S_{Y}\right)=\operatorname{dim} Y$ où $Y$ est un espace de Banach réel et $S_{Y}=\{u \in$ $\left.Y ;\|u\|_{Y}=1\right\}$.

3) $\theta(K)=0$ si et seulement si $K=\emptyset$.

4) S'il existe $\phi \in C\left(K_{1}, K_{2}\right)$ impaire, alors $\theta\left(K_{1}\right) \leqslant \theta\left(K_{2}\right)$. En particulier si $K_{1} \subset K_{2}$, alors $\theta\left(K_{1}\right) \leqslant \theta\left(K_{2}\right)$.

5) Si $K_{1}$ et $K_{2}$ sont homéomorphes par un homéomorphisme impaire, alors $\theta\left(K_{1}\right)=\theta\left(K_{2}\right)$.

6) Si $K$ est compact, alors $\theta(K)<+\infty$.

Preuve de la proposition 1.-1) Si $\theta(K)=k \in \mathbb{N}^{*}$. Alors il existe $f \in C\left(S^{k-1}, K\right)$ impaire, donc $k=\gamma\left(S^{k-1}\right) \leqslant \gamma(K)$.

Si $\theta(K)=+\infty$. Alors pour tout $k \in \mathbb{N}^{*}$ il existe $f \in C\left(S^{k-1}, K\right)$ impaire, donc $k=\gamma\left(S^{k-1}\right) \leqslant \gamma(K)$, par suite $\gamma(K)=+\infty$, d'où le résultat.

2) Si $0<\operatorname{dim} Y<+\infty$. Posons $n=\operatorname{dim} Y$ et soit $\left\{u_{1}, \ldots, u_{n}\right\}$ une base de $Y$ orthonormée. Définissons $f_{1}: S^{n-1} \rightarrow S_{Y}: f_{1}\left(\alpha_{1} \ldots, \alpha_{n}\right)=$ $\sum_{i=1}^{n} \alpha_{i} u_{i} \in S_{Y}$. Il est clair que l'application $f_{1}$ est un homéomorphisme continu impaire, donc $\theta\left(S_{Y}\right) \geqslant n$. D'autre part, supposons par l'absurde, que $\theta\left(S_{Y}\right)>n$, donc il existe $k>n$ et une application $f_{2}: S^{k-1} \rightarrow S_{Y}$ continue et impaire. Définissons $g=f_{1}^{-1} \circ f_{2}: S^{k-1} \rightarrow S^{n-1}$, alors $g$ est une application continue impaire définie de $S^{k-1}$ vers $S^{n-1}$ avec $k>n$, d'après le théorème de Borsuk, il existe $x \in S^{k-1}$ tel que $g(x)=g(-x)$, donc $\mathrm{g}(\mathrm{x})=0$, ce qui est absurde, d'où $\theta\left(S_{Y}\right)=n$.

Si $\operatorname{dim} Y=+\infty$, alors pour tout $n \geqslant 1$ il existe $X_{n}$ un sous espace vectoriel de $Y$ tel que $\operatorname{dim} X_{n}=n$. D'après le 1èr cas, on a $\theta\left(S_{Y} \cap X_{n}\right)=n$, par suite il existe une application $f: S^{n-1} \rightarrow S_{Y} \cap X_{n}$ continue impaire, donc $\theta\left(S_{Y}\right) \geqslant n$. Enfin, comme $n$ est arbitraire, on a $\theta\left(S_{Y}\right)=+\infty$.

La démonstration des assertions 3)...6) est similaire à celle du genre $\gamma$ (voir $[\mathrm{Ze}]$ ou $[\mathrm{Ch}]$ ).

Corollaire 1. - Soient $\Phi \in C^{1}(X, \mathbb{R})$ une fonctionnelle satisfaisant la condition (PS) sur $X, K_{0} \in S y m(X)$ un compact et $A \subset X$ un ensemble symétrique non vide. Si les conditions suivantes sont vérifiées

$\left(\mathrm{P}_{1}^{\prime}\right)$ Si $K \in \operatorname{Sym}(X)$ compact tel que $\theta(K) \geqslant \theta\left(K_{0}\right)+1$, alors $K \cap A \neq \emptyset$.

$\left(\mathrm{P}_{2}\right) \quad \alpha:=\max _{K_{0}} \Phi<\inf _{A} \Phi:=\beta$. 
Alors la valeur

$$
c:=\inf _{h \in \Gamma} \max _{u \in h(\bar{D})} \Phi(u)
$$

où $D=c o\left(K_{0}\right)$ et $\Gamma=\left\{h \in C(\bar{D}, X \backslash\{0\}) ; h=i d\right.$ sur $\left.K_{0}\right\}$ est une valeur critique de la fonctionnelle $\Phi$. De plus, on a

$$
c \geqslant \beta \text {. }
$$

Preuve du corollaire 1. - D'après le théorème 1 , il suffit de montrer que $h(\bar{D}) \cap A \neq \emptyset$ pour tout $h \in \Gamma$. Soit $h \in \Gamma$, posons $K=h(\bar{D}) \cup(-h(\bar{D}))$, il est clair que $K \in \operatorname{Sym}(X)$, compact et $K_{0} \subset K$. Ensuite, montrons que $\theta(K) \geqslant \theta\left(K_{0}\right)+1$. En effet. Posons $\theta(K)=n$. Alors Il existe une application $f_{1}: S^{n-1} \rightarrow K_{0}$ continue et impaire. Soit $\tilde{f}_{1}: \bar{D}_{n} \rightarrow c o\left(K_{0}\right)$ un prolongement continu de $f_{1}$ où $\bar{D}_{n}=\left\{x \in \mathbb{R}^{n} ;\|x\|_{\mathbb{R}^{n}} \leqslant 1\right\}$. Définissons l'application $f: S^{n} \rightarrow K$ par

$$
f\left(x_{1}, \ldots, x_{n}, x_{n+1}\right)=\left\{\begin{array}{lll}
h \circ \tilde{f}_{1}\left(x_{1}, \ldots, x_{n}\right) & \text { si } & x_{n+1} \geqslant 0 \\
-h \circ \tilde{f}_{1}\left(-x_{1}, \ldots,-x_{n}\right) & \text { si } & x_{n+1} \leqslant 0
\end{array}\right.
$$

Nous vérifions facilement que $f$ est bien défini, continue et impaire, donc $\theta(K) \geqslant \theta\left(K_{0}\right)+1$. L'hypothèse $\left(P_{1}^{\prime}\right)$ entraîne que $K \cap A \neq \emptyset$. Enfin, puisque $A$ est symétrique, il en résulte que $h(\bar{D}) \cap A \neq \emptyset$, d'où le corollaire.

\section{Remarques 2.}

1. Il existe dans la littérature plusieurs théorèmes de types minimax. Par exemples : Le théorème de liaisons[Z] et le théorème de point selle de Rabinowitz[Ra]. Ces théorèmes font intervenir la décomposition $V \oplus W=X$ où $V$ est un sous espace vectoriel de dimension finie. Le corollaire généralise ces théorèmes. En effet, soit $V \oplus W=X$ où $V$ et $W$ sont fermés et $\operatorname{dim} V<\infty$. On pose $K_{0}=\partial Q$ où $Q=\{u \in$ $V$ tel que $\|u\| \leqslant r$ \} pour un certain $r>0$, et $A=W$. D'après la proposition 1, on a d'une part $\theta\left(K_{0}\right)=\operatorname{dim} V$. D'autre part, on a le résultat général suivant :

Proposition 2. $-K \cap A \neq \emptyset$ pour tout $K \in \operatorname{Sym}(X)$ tel que $\gamma(K) \geqslant \theta\left(K_{0}\right)+1$.

Preuve de la proposition 2. - Soient $K \in \operatorname{Sym}(X)$ tel que $\gamma(K) \geqslant$ $\theta\left(K_{0}\right)+1$ et $P$ la projection de $X$ sur $V$. Supposons, par l'absurde, que $K \cap W=\emptyset$, alors $0 \notin P(K)$, par suite l'application $f=P_{/ K}$ : $K \rightarrow V \backslash\{0\} \simeq \mathbb{R}^{n} \backslash\{0\}$ est continue impaire, d'après la définition de $\gamma$, on a $\gamma(K) \leqslant n=\theta\left(K_{0}\right)$, ce qui est absurde. 
Sur un théorème de point critique et application à un problème de non-résonance

2. Les preuves du théorème de liaisons[Z] et du théorème de Rabinowitz[Ra] utilisent d'une manière essentielle la projection de $X$ sur $V$ afin de démontrer les conditions de liaisons. Ce qui n'est pas possible dans notre situation, d'où l'introduction des fonctions genre $\gamma$ et $\theta$.

3. La démonstration du corollaire contient le résultat suivant :

THÉORÈmE 2. - Soit $K_{0} \in \operatorname{Sym}(X)$ compact, on a $\theta(h(\bar{D}) \cup-h(\bar{D})) \geqslant \theta\left(K_{0}\right)+1$ pour tout application $h: \bar{D} \rightarrow X \backslash\{0\}$ continue telle que $h=i d$ sur $K_{0}$ où $D=c o\left(K_{0}\right)$.

4. L'hypothèse $: \operatorname{dim} X=+\infty$, intervient seulement pour démontrer qu'il existe $v \in X \backslash\{0\}$ tel que $D_{v} \cap K_{0}=\emptyset$ où $D_{v}=\{t v, t \geqslant 0\}$ et $K_{0}$ est un compact non vide tel que $0 \notin K_{0}$ (voir la lère étape du lemme 1). Si cette dernière condition est vérifiée, alors le théorème 1 reste vrai même lorsque l'espace $X$ est de dimension finie.

\section{Etude variationnelle du spectre du p-laplacien}

\subsection{Préliminaires et notations}

Soient $\Omega$ un domaine borné de $\mathbb{R}^{N}, 1<p<+\infty$ un réel, $\Delta_{p} u=\operatorname{div}\left(|\nabla u|^{p-2} \nabla u\right)$ le p-laplacien et $m \in M:=\left\{m \in L^{\infty}(\Omega) ; \operatorname{mes}\{x \in\right.$ $\Omega ; m(x)>0\} \neq 0\}$.

Rappelons que le spectre du p-laplacien dans $W_{0}^{1, p}(\Omega)$ avec poids $m$ est l'ensemble $\sigma\left(-\Delta_{p}\right)$ des $\lambda \in \mathbb{R}_{+}$pour lesquelles il existe une fonction $u$ (non triviale) solution de l'équation

$$
\left\{\begin{aligned}
-\Delta_{p} u & =\lambda m(x)|u|^{p-2} u & \text { dans } & \Omega \\
u & =0 & \text { sur } & \partial \Omega .
\end{aligned}\right.
$$

Ce spectre contient une suite $\left(\lambda_{n}(\gamma)\right)$ croissante tendant vers $+\infty$ qui a la caractérisation suivante :

$$
\frac{1}{\lambda_{n}(\gamma)}=\sup _{K \in A_{n}(\gamma)} \min _{u \in K} \int_{\Omega} m(x)|u|^{p}
$$

où $\gamma$ est la fonction genre et $A_{n}(\gamma)=\{K \subset S ; K$ compact, symétrique et $\gamma(K) \geqslant n\}$ avec $S=\left\{u \in W_{0}^{1, p}(\Omega) ;\|u\|_{1, p}:=\left(\int_{\Omega}|\nabla u|^{p}\right)^{\frac{1}{p}}=1\right\}$.

Des études particulières ont été faites sur la première et la deuxième valeur propre. Citons par exemple le résultat de simplicité et d'isolation de la première valeur propre [An1], et la caractérisation variationnelle de la deuxième valeur propre [An,Ts1]. 


\subsection{Une autre caractérisation variationnelle}

On considère la suite $\left(\lambda_{n}(\theta)\right)$ définie dans (4.2) en remplaçant $\gamma$ par $\theta$, on a :

THÉORÈmE 3 a) $\left(\lambda_{n}(\theta)\right)_{n}$ est une suite de valeurs propres du p-laplacien, croissante et tendant vers $+\infty$.

b) $\lambda_{1}:=\lambda_{1}(\theta)=\lambda_{1}(\gamma)$ ò̀ $\quad\left(\lambda_{1}\right)^{-1}=\sup _{u \in S} \int_{\Omega} m(x)|u|^{p}$.

c) $\lambda_{n}(\theta) \geqslant \lambda_{n}(\gamma)$ pour tout $n \geqslant 1$.

d) $\lambda_{2}:=\lambda_{2}(\theta)=\lambda_{2}(\gamma)$ (la deuxième valeur propre du p-laplacien).

e) Si $p=2$, alors $\lambda_{n}:=\lambda_{n}(\theta)=\lambda_{n}(\gamma)$ pour tout $n \geqslant 1$ et $\sigma(-\Delta)=$ $\left\{\lambda_{n}, n \geqslant 1\right\}$.

Preuve du théorème 3. - a) et $\mathrm{b}$ ) est une adaptation immédiate de la méthode développée dans [An2], pour plus de détail voir [Ch].

c) Provient du fait que $\theta \leqslant \gamma$.

d) D'après a) et c), on a $\lambda_{2}(\theta) \in \sigma\left(-\Delta_{p}\right)$ et $\lambda_{2}(\gamma) \leqslant \lambda_{2}(\theta)$. Il reste à montrer que $] \lambda_{1}, \lambda_{2}(\theta)\left[\cap \sigma\left(-\Delta_{p}\right)=\emptyset\right.$. Supposons, par l'absurde, qu'il existe $\lambda \in] \lambda_{1}, \lambda_{2}(\theta)\left[\cap \sigma\left(-\Delta_{p}\right)\right.$. Soit $u$ une fonction propre associée à $\lambda$, comme $\lambda \neq \lambda_{1}$, on a $u^{+} \not \equiv 0$ et $u^{-} \not \equiv 0$.

En multipliant le problème (4.1) respectivement par $u^{+}, u^{-}$et $u$, on obtient respectivement

$$
\begin{aligned}
\int_{\Omega}\left|\nabla u^{+}\right|^{p} & =\lambda \int_{\Omega} m(x)\left|u^{+}\right|^{p}, \\
\int_{\Omega}\left|\nabla u^{-}\right|^{p} & =\lambda \int_{\Omega} m(x)\left|u^{-}\right|^{p}, \\
\int_{\Omega}|\nabla u|^{p} & =\lambda \int_{\Omega} m(x)|u|^{p} .
\end{aligned}
$$

D'autre part, on désigne par $F_{2}$ le sous espace vectoriel de $W_{0}^{1, p}(\Omega)$ engendré par $u^{+}$et $u^{-}$, d'après la proposition 1 , on a $\theta\left(K_{2}\right)=2$ où $K_{2}=S \cap F_{2}$. Finalement, soit $a u^{+}+b u^{-} \in K_{2}$, on a

$$
\begin{gathered}
1=\left\|a u^{+}+b u^{-}\right\|_{1, p}^{p} \\
=|a|^{p}\left\|u^{+}\right\|_{1, p}^{p}+|b|^{p}\left\|u^{-}\right\|_{1, p}^{p}, \\
-16-
\end{gathered}
$$


Sur un théorème de point critique et application à un problème de non-résonance

combinée avec (4.3), donne

$$
\begin{aligned}
\int_{\Omega} m(x)\left|a u^{+}+b u^{-}\right|^{p} & =|a|^{p} \int_{\Omega} m(x)\left|u^{+}\right|^{p}+|b|^{p} \int_{\Omega} m(x)\left|u^{-}\right|^{p} \\
& =\frac{1}{\lambda}|a|^{p}\left\|u^{+}\right\|_{1, p}^{p}+\frac{1}{\lambda}|b|^{p}\left\|u^{-}\right\|_{1, p}^{p} \\
& =\frac{1}{\lambda}
\end{aligned}
$$

par conséquent

$$
\frac{1}{\lambda}=\min _{u \in K_{2}} \int_{\Omega} m(x)|u|^{p} \leqslant \frac{1}{\lambda_{2}(\theta)},
$$

de sorte que $\lambda_{2}(\theta) \leqslant \lambda$, ce qui est absurde.

e) Si $p=2$, on a $\sigma(-\Delta)=\left\{\lambda_{n}(\gamma), n \geqslant 1\right\}$. $\lambda_{n}(\gamma)$ est comptée avec son ordre de multiplicité (c'est-à-dire si $\lambda_{n-1}(\gamma)<\lambda_{n}(\gamma)=\ldots=\lambda_{n+q}(\gamma)<$ $\lambda_{n+q+1}(\gamma)$, alors $\operatorname{dim} E_{\lambda_{n}(\gamma)}=q+1$ où $E_{\lambda_{n}(\gamma)}$ est le sous espace propre associé à $\left.\lambda_{n}(\gamma)\right)$. Pour la preuve de ce résultat voir [Ch] ou [Az]. D'après $\mathrm{c}$ ) il reste à montrer que $\lambda_{n}(\theta) \leqslant \lambda_{n}(\gamma)$.

En effet, soit $\left\{\phi_{n}, n \geqslant 1\right\}$ une base Hilbertienne de $W_{0}^{1,2}(\Omega)$ où $\phi_{n}$ est une fonction propre du problème (4.1) associée à la valeur propre $\lambda_{n}(\gamma)$. Notons qu'on a aussi $\int_{\Omega} m(x) \phi_{i} \phi_{j}=0$ pour tout $i \neq j$. Posons $K_{n}=F_{n} \cap S$ où $F_{n}=\left\langle\phi_{1}, \ldots, \phi_{n}\right\rangle$ est le sous espace vectoriel de $W_{0}^{1,2}(\Omega)$ engendré par les fonctions $\phi_{1}, \phi_{2}, \ldots$ et $\phi_{n}$. D'après la proposition 1 , on a $\theta\left(K_{n}\right)=n$, donc $K_{n} \in A_{n}(\theta)$, il en résulte que

$$
\min _{u \in K_{n}} \int_{\Omega} m(x)|u|^{2} \leqslant \frac{1}{\lambda_{n}(\theta)} .
$$

D'autre part, soit $u=\sum_{i=1}^{n} \alpha_{i} \phi_{i} \in K_{n}$, on a

$$
\begin{aligned}
1 & =\|u\|_{1,2}^{2} \\
& =\left\|\sum_{i=1}^{n} \alpha_{i} \phi_{i}\right\|_{1,2}^{2} \\
& =\sum_{i=1}^{n}\left|\alpha_{i}\right|^{2}\left\|\phi_{i}\right\|_{1,2}^{2} \\
& =\sum_{i=1}^{n} \lambda_{i}(\gamma)\left|\alpha_{i}\right|^{2} \int_{\Omega} m(x)\left|\phi_{i}\right|^{2} \\
& \leqslant \lambda_{n}(\gamma) \sum_{i=1}^{n}\left|\alpha_{i}\right|^{2} \int_{\Omega} m(x)\left|\phi_{i}\right|^{2} \\
& =\lambda_{n}(\gamma) \int_{\Omega} m(x)|u|^{2},
\end{aligned}
$$


par conséquent

$$
\frac{1}{\lambda_{n}(\gamma)}=\min _{u \in K_{n}} \int_{\Omega} m(x)|u|^{2} \leqslant \frac{1}{\lambda_{n}(\theta)}
$$

Ce qui prouve le point e).

Dans le cas $N=1$ et $m \equiv 1$, Otani ([Ot1] et [Ot2]) a déterminé entièrement le spectre $\sigma\left(-\Delta_{p}\right)$ et a donné une caractérisation complète des fonctions propres. Plus précisément, si on prend $\Omega=] 0, \pi[$, on a

Proposition 3 ([Ot1] et [Ot2]).

i) Le spectre $\sigma\left(-\Delta_{p}\right)$ est une suite $\left(\mu_{n}\right)_{n}$ de nombre réels $0<\mu_{1}<\mu_{2}<$ $\ldots<\mu_{k} \ldots$ où $\mu_{n}=n^{p} \mu_{1}$ avec $\mu_{1}=(p-1)\left\{\frac{1}{\pi} \int_{0}^{1} \frac{d t}{\left(1-t^{p}\right)^{\frac{1}{p}}}\right\}^{p}$.

ii) Pour tout $n \geqslant 1$, la valeur propre $\mu_{n}$ est simple et la fonction propre associée $u_{n}$ admet $(n-1)$ zéros distincts sur $] 0, \pi[$.

Il est bien clair, d'après le théorème 3 , que $\mu_{1}=\lambda_{1}(\theta)=\lambda_{1}(\gamma)$ et $\mu_{2}=\lambda_{2}(\theta)=\lambda_{2}(\gamma)$.

Il est naturel de se poser la question de savoir si la suite donnée par (4.2) coïncide avec le spectre $\sigma\left(-\Delta_{p}\right)$. La réponse est affirmative, et nous avons :

THÉORÈme 4. - Pour tout $n \geqslant 1$, on a $\mu_{n}=\lambda_{n}(\theta)=\lambda_{n}(\gamma)$, de plus, il existe $K_{n} \in \operatorname{Sym}\left(W_{0}^{1, p}(] 0, \pi[)\right)$ compact tel que $\theta\left(K_{n}\right)=\gamma\left(K_{n}\right)=n$ et $\frac{1}{\mu_{n}}=\min _{u \in K_{n}} \int_{0}^{\pi}|u|^{p}$.

Preuve $d u$ théorème 4. $-\mathbf{1}^{\text {re }}$ étape, montrons que $\lambda_{n}(\theta) \leqslant \mu_{n}$ pour tout $n \geqslant 1$.

Soit $n \geqslant 3$ et $u_{n}$ une fonction propre associée à $\mu_{n}$. Désignons par $0, t_{1}, \ldots, t_{n-1}, \pi$ les zéros de la fonction $u_{n}$, tels que $0<t_{1}<\cdots<t_{n-1}<\pi$, puis définissons

$$
v_{i}(x)=\left\{\begin{array}{ccc}
u_{n}(x) & \text { si } & x \in\left[t_{i-1}, t_{i}\right] \\
0 & \text { si } & x \notin\left[t_{i-1}, t_{i}\right]
\end{array}\right.
$$

pour tout $i=1,2, \ldots, n$ où $t_{0}=0$ et $t_{n}=\pi$. Il est clair que $v_{i} \in W_{0}^{1, p}(] 0, \pi[)$ pour tout $1 \leqslant i \leqslant n$. En multipliant l'équation (4.1) par $v_{i}$, on obtient

$$
\int_{0}^{\pi}\left|v_{i}^{\prime}\right|^{p}=\mu_{n} \int_{0}^{\pi}\left|v_{i}\right|^{p}
$$


Sur un théorème de point critique et application à un problème de non-résonance

On pose $K_{n}=F_{n} \cap S$ où $F_{n}=\left\langle v_{1}, \ldots, v_{n}\right\rangle$ est le sous espace vectoriel de $W_{0}^{1, p}(] 0, \pi[)$ engendré par $v_{1}, \ldots, v_{n}$. Par la proposition 1 , on a $\theta\left(K_{n}\right)=n$. On sait aussi que $\gamma\left(K_{n}\right)=n$. Enfin, soit $u=\sum_{i=1}^{n} \alpha_{i} v_{i} \in K_{n}$, en tenant compte de (4.4), il vient que

$$
\begin{aligned}
1 & =\int_{0}^{\pi}\left|u^{\prime}\right|^{p} \\
& =\sum_{i=1}^{n}\left|\alpha_{i}\right|^{p} \int_{0}^{\pi}\left|v_{i}^{\prime}\right|^{p} \\
& =\mu_{n} \sum_{i=1}^{n}\left|\alpha_{i}\right|^{p} \int_{0}^{\pi}\left|v_{i}\right|^{p} \\
& =\mu_{n} \int_{0}^{\pi}|u|^{p}
\end{aligned}
$$

donc $\frac{1}{\mu_{n}}=\min _{u \in K_{n}} \int_{0}^{\pi}|u|^{p}$, il en résulte (d'après (4.2)) que $\frac{1}{\mu_{n}} \leqslant \frac{1}{\lambda_{n}(\theta)}$, ou encore $\lambda_{n}(\theta) \leqslant \mu_{n}$. Ce qui prouve la lère étape.

$2^{\mathrm{e}}$ étape. D'après la lère étape et comme $\lambda_{n}(\gamma) \leqslant \lambda_{n}(\theta)$, il reste à montrer que $\lambda_{n}(\gamma)=\mu_{n}$. Raisonnons par récurrence. Pour $n=1,2$ le résultat est vrai. Ensuite, supposons que $\lambda_{n}(\gamma)=\mu_{n}$. Puisque les fonctions propres sont simples (proposition 3), alors $\lambda_{n}(\gamma)<\lambda_{n+1}(\gamma)([\mathrm{Ze}])$, or $\lambda_{n}(\gamma)=\mu_{n}$ et $] \mu_{n}, \mu_{n+1}\left[\cap \sigma\left(\Delta_{p}\right)=\emptyset\right.$, donc $\mu_{n+1} \leqslant \lambda_{n+1}(\gamma)$, par suite, la lère étape et la proposition 1 impliquent que $\lambda_{n+1}(\gamma) \leqslant \lambda_{n+1}(\theta) \leqslant \mu_{n+1}$. Ceci termine la démonstration du théorème.

\section{Condition de Palais-Smale}

Considérons le problème de Dirichlet

$$
\left\{\begin{aligned}
-\Delta_{p} u & =f(x, u)+h & \text { dans } & \Omega \\
u & =0 & \text { sur } & \partial \Omega
\end{aligned}\right.
$$

où $f: \Omega \times \mathbb{R} \rightarrow \mathbb{R}$ est une fonction de Carathéodory et $h \in W^{-1, p^{\prime}}(\Omega)$. Nous supposons dans toute la suite que la fonction $f$ satisfait l'hypothèse

$$
\forall R>0, \quad \exists \phi_{R} \in L^{p^{\prime}}(\Omega) \text { tel que } \max _{|s| \leqslant R}|f(x, s)| \leqslant \phi_{R}(x) \text { p.p. } x \in \Omega .
$$

Soient $\alpha, \beta$ des réels tels que $\alpha<\beta$, on considère les hypothèses :

$\left(\mathbf{f}_{\alpha, \beta}\right) \quad \forall \varepsilon>0, \exists b_{\varepsilon} \in L^{p^{\prime}}(\Omega)$ tel que p.p. $x \in \Omega, \forall s \in \mathbb{R}$, on a

$$
-b_{\varepsilon}(x)+(\alpha-\varepsilon)|s|^{p} \leqslant s f(x, s) \leqslant(\beta+\varepsilon)|s|^{p}+b_{\varepsilon}(x) .
$$


$\left(\mathbf{F}_{\alpha, \beta}\right) \quad \alpha \leqslant l \equiv l(x):=\liminf _{s \rightarrow \pm \infty} \frac{p F(x, s)}{|s|^{p}}, \limsup _{s \rightarrow \pm \infty} \frac{p F(x, s)}{|s|^{p}}:=k(x) \leqslant \beta$ p.p. $x \in \Omega$ et

$$
\begin{aligned}
& \forall \varepsilon>0, \exists d_{\varepsilon} \in L^{1}(\Omega) \text { tel que p.p. } x \in \Omega, \forall s \in \mathbb{R}, \text { on a } \\
& -d_{\varepsilon}(x)+(l(x)-\varepsilon) \frac{|s|^{p}}{p} \leqslant F(x, s) \leqslant(k(x)+\varepsilon) \frac{|s|^{p}}{p}+d_{\varepsilon}(x)
\end{aligned}
$$

où $F: \Omega \times \mathbb{R} \rightarrow \mathbb{R}$ est une fonction définie par $F(x, s)=\int_{0}^{s} f(x, t) d t$.

La notation $\leqslant \not \equiv$ signifie qu'on a une inégalité large p.p. sur $\Omega$ et stricte sur un sous ensemble de $\Omega$ de mesure non nulle.

Nous associons au problème (5.1) la fonctionnelle énergie

$$
\Phi_{p}(u)=\frac{1}{p} \int_{\Omega}|\nabla u|^{p}-\int_{\Omega} F(x, u)-\langle h, u\rangle
$$

Remarques 3

1. L'hypothèse $\left(f_{\alpha, \beta}\right)$ signifie :

$$
\alpha \leqslant \liminf _{s \rightarrow \pm \infty} \frac{f(x, s)}{|s|^{p-2} s} \text { et } \limsup _{s \rightarrow \pm \infty} \frac{f(x, s)}{|s|^{p-2} s} \leqslant \beta, \quad\left(f_{\alpha, \beta}\right)
$$

avec une certaine uniformité en $x \in \Omega$.

2. Si on suppose (5.2) et $\left(f_{\alpha, \beta}\right)$, alors il existe un réel $a>0$ et une fonction $d \in L^{p^{\prime}}(\Omega)$ tels que

$$
|f(x, s)| \leqslant a|s|^{p-1}+d(x) \quad \text { p.p. } x \in \Omega, \forall s \in \mathbb{R},
$$

par suite la fonctionnelle $\Phi_{p}$ est bien définie sur $W_{0}^{1, p}(\Omega)$, elle est de classe $C^{1}$ et sa dérivée de Frêchet en tout point $u \in W_{0}^{1, p}(\Omega)$ est : $\Phi_{p}^{\prime}(u)=-\Delta_{p} u-f(x, u)-h$.

3. L'hypothèse $\left(F_{\alpha, \beta}\right)$ est satisfaite dès que la fonction $f$ vérifie la condition $\left(f_{\alpha, \beta}\right)$ en remplaçant $\leqslant$ par $\leqslant \not \equiv$. Mais on démontre facilement que l'inverse est généralement faux.

Dans cette section, nous allons examiner des situations dans lesquelles la fonctionnelle $\Phi_{p}$ satisfait la condition de Palais-Smale (PS).

LEMME 2. - On suppose (5.2), $\left(f_{\alpha, \beta}\right)$ et $\left(F_{\alpha, \beta}\right)$. Si la fonctionnelle $\Phi_{p}$ ne satisfait pas la condition (PS), alors il existe $m(x) \in L^{\infty}(\Omega), v \in$ 
Sur un théorème de point critique et application à un problème de non-résonance

$W_{0}^{1, p}(\Omega) \backslash\{0\}$ et $\left(u_{n}\right) \subset W_{0}^{1, p}(\Omega)$ tels que $v$ est une solution non triviale $d u$ problème

$$
\left\{\begin{aligned}
-\Delta_{p} u & =m|u|^{p-2} u & \text { dans } & \Omega \\
u & =0 & \text { sur } & \partial \Omega
\end{aligned}\right.
$$

et

$$
\left\{\begin{array}{l}
\alpha \leqslant \not \equiv m(x) \leqslant \not \equiv \beta \text { p.p. } x \in \Omega, \\
\left\|u_{n}\right\|_{1, p} \rightarrow+\infty, \quad \frac{u_{n}}{\left\|u_{n}\right\|_{1, p}} \rightarrow v \text { dans } W_{0}^{1, p}(\Omega) \\
\left(\Phi_{p}\left(u_{n}\right)\right)_{n} \quad \text { est une suite bornée. }
\end{array}\right.
$$

La démonstration est analogue à celle du cas linéaire $p=2$ (voir par exemple $[\mathrm{Co}, \mathrm{Ol}]$ ) avec une légère adaptation, comme par exemple, dans le passage à la limite d'une suite de (PS), on utilise le fait que l'opérateur $-\Delta_{p}$ est de type $\left(S_{+}\right)$. (Pour plus de détaille voir [Ch]).

Soit $m \in M$ où $M$ est l'ensemble défini dans la section 4. S'il y a risque de confusion on note par: $\lambda_{n}(m)=\lambda_{n}, \lambda_{n}(\theta, m)=\lambda_{n}(\theta), \lambda_{n}(\gamma, m)=\lambda_{n}(\gamma)$ et $\sigma\left(-\Delta_{p}, m\right)=\sigma\left(-\Delta_{p}\right)$.

Définition 3. - On dit que le problème $\left(P_{m}\right)$ satisfait la propriété de la continuation unique (P.C.U), si pour toute solution $u$ de $\left(P_{m}\right)$ telle que $m e s\{x \in \Omega ; u(x)=0$ p.p $\} \neq 0$, alors $u=0$ p.p. sur $\Omega$.

Il existe une large littérature traitant des problèmes de continuation unique : voir à ce sujet par exemple [De], [De,Go], [Go,Lo] et [Lo].

Rappelons que dans le cas linéaire $p=2$, le problème $\left(P_{m}\right)$ satisfait la condition (P.C.U) pour tout $m \in M$ (voir [Go,Lo] ou [De]).

Toujours dans le cas $p=2$, De Figueiredo et Gossez ont montré dans $[\mathrm{De}, \mathrm{Go}]$ la proposition suivante :

Proposition 4. - Si $p=2$, alors le problème $\left(P_{m}\right)$ vérifie (P.C.U) si et seulement si $\lambda_{n}(m)$ est strictement monotone par rapport au poids $m$ pour tout $n$ (c'est-à-dire si $m_{1}, m_{2} \in M$ tels que $m_{1} \leqslant \not \equiv m_{2}$, alors $\lambda_{n}\left(m_{1}\right)>\lambda_{n}\left(m_{2}\right)$ pour tout $\left.n\right)$.

Une conséquence de cette proposition est que si $\lambda_{n} \leqslant \not \equiv m(x) \leqslant \not \equiv \lambda_{n+1}$ p.p. $x \in \Omega$, alors le problème $\left(P_{m}\right)$ n'admet pas de solution non triviale.

Dans le cas général $p \neq 2$, un résultat de stricte monotonie a été établi par Anane et Tsouli dans [An,Ts1]. Il ont montré la stricte monotonie de $\lambda_{1}(m)$ par rapport au poids et la stricte monotonie partielle de $\lambda_{2}(m)$ par rapport au poids dans le sens que : Si $m_{1}, m_{2} \in M$ tels que $m_{1}<m_{2}$ p.p. $x \in \Omega$, alors $\lambda_{2}\left(m_{2}\right)<\lambda_{2}\left(m_{1}\right)$. 
Comme conséquence de ce résultat, on a

Proposition 5. - Pour tout $1<p<+\infty$, si $\lambda_{1}(1) \leqslant \not \equiv m(x)<\lambda_{2}(1)$ p.p. $x \in \Omega$, alors le problème $\left(P_{m}\right)$ n'admet pas de solution non triviale.

Cette proposition est établie dans [An,Ts1]. Nous avons aussi la proposition suivante :

Proposition 6. - Soit $1<p<+\infty$.

1) Si $\lambda_{1}(1) \leqslant \not \equiv m(x) \leqslant \lambda_{2}(1)$ p.p. $x \in \Omega$ et $u$ une solution non triviale de $\left(P_{m}\right)$, alors $m(x)=\lambda_{2}(1)$ p.p. sur $\{x \in \Omega ; u(x) \neq 0\}$ et $u$ est une solution du problème $\left(P_{\lambda_{2}(1)}\right)$.

2) Si $\lambda_{1}(1) \leqslant \equiv \equiv(x)<\lambda_{2}(1)$ p.p. $x \in \Omega$, ou si

$$
\left\{\begin{array}{l}
\lambda_{1}(1) \leqslant \equiv \equiv(x) \leqslant \equiv \lambda_{2}(1) \\
\left(P_{\lambda_{2}(1)}\right) \text { satisfait }(\text { P.C.U) }
\end{array}\right.
$$

alors $\left(P_{m}\right)$ n'admet pas de solution non triviale et $\lambda_{1}(m)<1<\lambda_{2}(m)$.

Preuve de la proposition 6. - 1) C'est un résultat établi dans [Cu,De,Go].

2) Il est clair, d'après la stricte monotonie de $\lambda_{1}(m)$, que $\lambda_{1}(m)<$ $\lambda_{1}\left(\lambda_{1}(1)\right)=1$. Si $m(x)<\lambda_{2}(1)$ p.p. $x \in \Omega$, par la proposition $5,\left(P_{m}\right)$ n'admet pas de solution non triviale, et d'après la stricte monotonie partielle de $\lambda_{2}(m)$ [An,Ts2], on déduit $\lambda_{2}(m)>\lambda_{2}\left(\lambda_{2}(1)\right)=1$.

Finalement, si la condition (5.5) est vérifiée, alors d'après l'assertion 1 de cette proposition, $\left(P_{m}\right)$ n'admet pas de solution non triviale. Il en résulte que $1<\lambda_{2}(m)$, car sinon, on aura $1=\lambda_{2}(m)$, donc une fonction propre associée à $\lambda_{2}(m)=1$ est une solution non triviale du problème $\left(P_{m}\right)$, ce qui sera absurde.

Dans le cas $N=1$, nous avons la proposition suivante :

Proposition 7.- Supposons que $N=1$, soient $m \in L^{\infty}(\Omega)$ et $1<$ $p<+\infty$.

1) Si $\mu_{n} \leqslant \not \equiv m(x) \leqslant \not \equiv \mu_{n+1}$ p.p. $\left.x \in\right] 0, \pi[$ pour un certain entier $n \geqslant 1$, alors le problème $\left(P_{m}\right)$ n'admet pas de solution non triviale.

2) Si $\mu_{n} \leqslant \not \equiv m(x) \leqslant \not \equiv \mu_{n+1}$ p.p. $\left.x \in\right] 0, \pi\left[\right.$, alors $\lambda_{n}(\theta, m)<1<$ $\lambda_{n+1}(\gamma, m)$. Par suit $\lambda_{n}(\theta, m)<1<\lambda_{n+1}(\theta, m)$.

Preuve de la proposition 7. - 1) Voir [Pi,Ma]. 
Sur un théorème de point critique et application à un problème de non-résonance

2) Montrons que $\lambda_{n}(\theta, m)<1$ (démonstration analogue pour $\left.1<\lambda_{n+1}(\gamma, m)\right)$.

D'après (4.2), on a

$$
\begin{aligned}
\frac{1}{\lambda_{n}(\theta, m)} & =\sup _{K \in A_{n}(\theta)} \min _{u \in K} \int_{\Omega} m(x)|u|^{p} \\
& \geqslant \sup _{K \in A_{n}(\theta)} \min _{u \in K} \int_{\Omega} \mu_{n}|u|^{p} \\
& =\mu_{n} \sup _{\min _{n}(\theta)} \min _{u \in K}|u|_{\Omega}^{p} \\
& =\frac{\mu_{n}}{\lambda_{n}(\theta, 1)} .
\end{aligned}
$$

Puisque, d'après le théorème 4 , on a $\mu_{n}=\lambda_{n}(\theta, 1)$, il en résulte que $\lambda_{n}(\theta, m) \leqslant 1$.

D'autre part, supposons, par l'absurde, que $\lambda_{n}(\theta, m)=1$, donc $1 \in$ $\sigma\left(-\Delta_{p}, m\right)$, par suite le problème $\left(P_{m}\right)$ admet une solution non triviale, ce qui est en contradiction avec l'assertion 1 de cette proposition.

Dans la suite et s'il $\mathbf{n}$ y a pas risque de confusion, nous désignerons par $\lambda_{n}:=\lambda_{n}(1)$ la $n$ ième valeur propre du problème (4.1) avec poids $m=1$.

THÉORÈME 5. - La fonctionnelle $\Phi_{p}$ satisfait la condition (PS) dans les cas suivants :

1) $N=1,1<p<+\infty$ et la fonction $f$ satisfait $\left(f_{\mu_{n}, \mu_{n+1}}\right)$ et $\left(F_{\mu_{n}, \mu_{n+1}}\right)$ pour un certain entier $n \geqslant 1$.

2) $N>1,1<p<+\infty$, le problème $\left(P_{\lambda_{2}(1)}\right)$ satisfait la condition (P.C.U.) et la fonction $f$ satisfait $\left(F_{\lambda_{1}, \lambda_{2}}\right)$ et $\left(f_{\lambda_{1}, \lambda_{2}}\right)$.

3) $N>1,1<p<+\infty$ et $f$ satisfait $\left(F_{\lambda_{1}, \lambda_{2}}\right)$ et $\left(f_{\lambda_{1}, \lambda_{2}}\right)$ avec $k(x)<\lambda_{2}$ p.p.x $\in \Omega$.

4) $N>1,1<p<+\infty$ et $f(x, s)=\lambda m(x)|s|^{p-2} s$ où $m \in M$ et tel que $\lambda \notin \sigma\left(-\Delta_{p}, m\right)$.

5) $N>1, p=2$ et la fonction $f$ satisfait $\left(f_{\lambda_{n}, \lambda_{n+1}}\right)$ et $\left(F_{\lambda_{n}, \lambda_{n+1}}\right)$ pour un certain $n \geqslant 1$.

Preuve du théorème 5. - 1) Supposons, par l'absurde, que $\Phi_{p}$ ne satisfait pas la condition (PS).

D'après le lemme 2 , il existe $m \in L^{\infty}(\Omega)$ tel que le problème $\left(P_{m}\right)$ admet une solution non triviale et $\mu_{n} \leqslant \not \equiv m(x) \leqslant \not \equiv \mu_{n+1}$, ce qui est contradictoire avec la proposition 7 . 
2) Si $\Phi_{p}$ ne satisfait pas la condition (PS), alors le lemme 2 entraîne qu'il existe $m \in L^{\infty}(\Omega)$ tel que $\lambda_{1}(1) \leqslant \not \equiv m(x) \leqslant \not \equiv \lambda_{2}(1)$ et $v \in W_{0}^{1, p}(\Omega) \backslash\{0\}$ solution du problème $\left(P_{\lambda_{2}(1)}\right)$, puisque nous avions supposé la (P.C.U), il en résulte, d'après la proposition 6 , que $\lambda_{2}(1)=m(x)$ p.p. $x \in \Omega$, ce qui est absurde, d'où l'assertion 2.

3) Si $\Phi_{p}$ ne satisfait pas la condition (PS), alors il existe $m(x) \in L^{\infty}(\Omega)$, $v \in W_{0}^{1, p}(\Omega) \backslash\{0\}$ et $\left(u_{n}\right) \subset W_{0}^{1, p}(\Omega)$ vérifiant les conditions du lemme 2 (pour $\alpha=\lambda_{1}(1)$ et $\beta=\lambda_{2}(1)$ ). D'après la proposition $6, v$ est une solution du problème $P_{\lambda_{2}(1)}$, donc

$$
\begin{aligned}
\lambda_{2}(1) \int_{\Omega}|v|^{p} & =\int_{\Omega}|\nabla v|^{p} \\
& =\lim _{n \rightarrow+\infty} \int_{\Omega} \frac{F\left(x, u_{n}\right)}{\left\|u_{n}\right\|_{1, p}^{p}},
\end{aligned}
$$

il vient, d'après $\left(F_{\lambda_{1}, \lambda_{2}}\right)$, que

$$
\lambda_{2}(1) \int_{\Omega}|v|^{p} \leqslant \int_{\Omega}(k(x)+\varepsilon)|v|^{p} .
$$

Comme $\varepsilon$ est arbitraire, on déduit que $\left(k(x)-\lambda_{2}(1)\right)|v|^{p}=0$ p.p. $x \in \Omega$. Enfin, puisque $k(x)<\lambda_{2}(1)$ p.p., il en résulte que $v=0$, ce qui est absurde, d'où l'assertion 3 .

4) Supposons, par l'absurde, que $\Phi_{p}$ ne satisfait pas la condition (PS). On peut, sans perte de généralité, supposer qu'il existe une suite $\left(u_{n}\right) \subset$ $W_{0}^{1, p}(\Omega)$ telle que a) $\left\|u_{n}\right\|_{1, p} \rightarrow+\infty$, b) $\left(\Phi_{p}\left(u_{n}\right)\right)$ est bornée, et c) $\Phi_{p}^{\prime}\left(u_{n}\right) \rightarrow$ 0 . Posons $v_{n}=\frac{u_{n}}{\left\|u_{n}\right\|_{1, p}}$, il existe une sous suite de $v_{n}$ (notée aussi $v_{n}$ ) telle que $v_{n} \rightarrow v$ dans $L^{p}(\Omega)$ et $v_{n} \rightarrow v$ dans $W_{0}^{1, p}(\Omega)$. En divisons $\Phi_{p}^{\prime}\left(u_{n}\right)$ par $\left\|u_{n}\right\|_{1, p}^{p-1}$, on obtient

$$
v_{n}=\left(-\Delta_{p}\right)^{-1}\left(\lambda m\left|v_{n}\right|^{p-2} v_{n}+\frac{h}{\left\|u_{n}\right\|_{1, p}^{p-1}}+\frac{\Phi_{p}^{\prime}\left(u_{n}\right)}{\left\|u_{n}\right\|_{1, p}^{p-1}}\right) .
$$

Il est clair que $\lambda m\left|v_{n}\right|^{p-2} v_{n}+\frac{h}{\left\|u_{n}\right\|_{1, p}^{p-1}}+\frac{\Phi_{p}^{\prime}\left(u_{n}\right)}{\left\|u_{n}\right\|_{1, p}^{p-1}} \rightarrow \lambda m|v|^{p-2} v$ dans $W^{-1, p^{\prime}}(\Omega)$, puisque $-\Delta_{p}: W_{0}^{1, p}(\Omega) \rightarrow W^{-1, p^{\prime}}(\Omega)$ est un homéomorphisme, il vient que

$v_{n} \rightarrow v$ dans $W_{0}^{1, p}(\Omega)$ et $-\Delta_{p} v=\lambda m|v|^{p-2} v$ dans $W^{-1, p^{\prime}}(\Omega)$, de sorte que $v \not \equiv 0$ et $\lambda \in \sigma\left(-\Delta_{p}, m\right)$, ce qui est absurde.

5) Même démonstration que 1), dans ce cas on utilise le lemme 2 et la proposition 4 . 
Sur un théorème de point critique et application à un problème de non-résonance

\section{Applications}

THÉORÈME 6. - Dans les situations suivantes, la fonctionnelle $\Phi_{p}$ admet au moins un point critique dont la valeur critique est caractérisée par un principe de minimax

1) $N=1,1<p<+\infty$ et la fonction $f$ satisfait $\left(f_{\mu_{n}, \mu_{n+1}}\right)$ et $\left(F_{\mu_{n}, \mu_{n+1}}\right)$ pour un certain entier $n \geqslant 1$.

2) $N>1,1<p<+\infty$, le problème $\left(P_{\lambda_{2}(1)}\right)$ satisfait la condition (P.C.U.) et la fonction $f$ satisfait $\left(F_{\lambda_{1}, \lambda_{2}}\right)$ et $\left(f_{\lambda_{1}, \lambda_{2}}\right)$.

3) $N>1,1<p<+\infty$ et $f$ satisfait $\left(F_{\lambda_{1}, \lambda_{2}}\right)$ et $\left(f_{\lambda_{1}, \lambda_{2}}\right)$ avec $k(x)<\lambda_{2}$ p.p.x $\in \Omega$.

4) $N>1,1<p<+\infty$ et $f(x, s)=\lambda m(x)|s|^{p-2} s$ où $m \in M$ et tel que $\lambda \notin \sigma\left(-\Delta_{p}, m\right)$.

5) $N>1, p=2$ et la fonction $f$ satisfait $\left(f_{\lambda_{n}, \lambda_{n+1}}\right)$ et $\left(F_{\lambda_{n}, \lambda_{n+1}}\right)$ pour un certain $n \geqslant 1$.

Preuve du théorème 6. - Ces résultats sont des applications du corollaire 1 . Nous allons montrer que les hypothèses de ce corollaire sont satisfaites.

1) D'après le théorème 5 , la fonctionnelle $\Phi_{p}$ satisfait la condition (PS). Soient $l(x)$ et $k(x)$ les fonctions qui interviennent dans l'hypothèse $\left(F_{\mu_{n}, \mu_{n+1}}\right)$ et posons

$$
A=\left\{u \in W_{0}^{1, p}(] 0, \pi[) ; \lambda_{n+1}(\theta, k) \int_{0}^{\pi} k(x)|u|^{p} \leqslant \int_{0}^{\pi}\left|u^{\prime}\right|^{p}\right\}
$$

où $\lambda_{n+1}(\theta, k)$ est la valeur propre définie par (4.2). D'autre part, soit $\varepsilon>0$, d'après (4.2) et le théorème 3 , il existe $K^{\prime} \in A_{n}(\theta)$ tel que

$$
\frac{1}{\lambda_{n}(\theta, l)}-\varepsilon \leqslant \min _{u \in K^{\prime}} \int_{0}^{\pi} l(x)|u|^{p} .
$$

Montrons que : i) $\Phi_{p}(u) \rightarrow-\infty$ lorsque $\|u\|_{1, p} \rightarrow+\infty$ et $u \in \mathbb{R} K^{\prime}$. ii) $\Phi_{p}$ est coercive sur $A$ (c-à-d $\Phi_{p}(u) \rightarrow+\infty$ lorsque $\|u\|_{1, p} \rightarrow+\infty$ et $u \in A$ ).

En effet : Pour tout $u \in W_{0}^{1, p}(] 0, \pi[)$, d'après $\left(F_{\mu_{n}, \mu_{n+1}}\right)$, on a

$$
\Phi_{p}(u) \leqslant \frac{1}{p}\|u\|_{1, p}^{p}-\frac{1}{p} \int_{0}^{\pi}(l(x)-\varepsilon)|u|^{p}+\int_{0}^{\pi} d_{\varepsilon}(x)+\|u\|_{1, p}\|h\|_{-1, p^{\prime}}
$$


et

$$
\Phi_{p}(u) \geqslant \frac{1}{p}\|u\|_{1, p}^{p}-\frac{1}{p} \int_{0}^{\pi}(k(x)+\varepsilon)|u|^{p}-\int_{0}^{\pi} d_{\varepsilon}(x)-\|u\|_{1, p}\|h\|_{-1, p^{\prime}} .
$$

Si $u \in \mathbb{R} K^{\prime}$, alors par (6.1), (6.2) et le fait que $\int_{0}^{\pi}|u|^{p} \leqslant \frac{\|u\|_{1, p}^{p}}{\lambda_{1}(1)}$, on obtient

$$
\begin{aligned}
\Phi_{p}(u) & \leqslant \frac{1}{p}\left(1-\frac{1}{\lambda_{n}(\theta, l)}\right)\|u\|_{1, p}^{p}+\frac{\varepsilon}{p} \int_{0}^{\pi}|u|^{p}+\int_{0}^{\pi} d_{\varepsilon}(x)+\|u\|_{1, p}\|h\|_{-1, p^{\prime}} \\
& \leqslant \frac{1}{p} \mu\|u\|_{1, p}^{p}+\int_{0}^{\pi} d_{\varepsilon}(x)+\|u\|_{1, p}\|h\|_{-1, p^{\prime}}
\end{aligned}
$$

où $\mu=\left(1-\frac{1}{\lambda_{n}(\theta, l)}+\frac{\varepsilon}{\lambda_{1}(1)}\right)$. D'après la proposition 7 , on a $1-\frac{1}{\lambda_{n}(\theta, l)}<0$, donc pour $\varepsilon$ assez petit tel que $\mu<0$, l'affirmation i) est vérifiée. D'autre part, si $u \in A$, alors (6.3) implique

$$
\begin{aligned}
\Phi_{p}(u) & \geqslant \frac{1}{p}\left(1-\frac{1}{\lambda_{n+1}(\theta, k)}\right)\|u\|_{1, p}^{p}-\frac{\varepsilon}{p} \int_{0}^{\pi}|u|^{p}-\int_{0}^{\pi} d_{\varepsilon}(x)-\|u\|_{1, p}\|h\|_{-1, p^{\prime}} \\
& \geqslant \frac{1}{p} \mu^{\prime}\|u\|_{1, p}^{p}-\int_{0}^{\pi} d_{\varepsilon}(x)-\|u\|_{1, p}\|h\|_{-1, p^{\prime}}
\end{aligned}
$$

où $\mu^{\prime}=\left(1-\frac{1}{\lambda_{n+1}(\theta, k)}-\frac{\varepsilon}{\lambda_{1}(1)}\right)$. D'après la proposition 7 , on a $1-$ $\frac{1}{\lambda_{n+1}(\theta, k)}>0$, donc l'affirmation ii) est vérifiée pour $\varepsilon$ assez petit tel que $\mu^{\prime}>0$. Finalement, d'après l'affirmation ii) la valeur $\beta:=\inf _{u \in A} \Phi_{p}(u)$ est finie. Comme $K^{\prime}$ est compact et par l'affirmation i), il existe $t_{0}$ assez grand tel que $\alpha:=\max _{u \in t_{0} K^{\prime}} \Phi_{p}(u)<\beta$. Ensuite, posons $K_{0}=t_{0} K^{\prime}$, on a $K_{0} \in \operatorname{Sym}\left(W_{0}^{1, p}(] 0, \pi[)\right), K_{0}$ est compact et que $\theta\left(K_{0}\right) \geqslant n$, donc l'hypothèse $\left(P_{2}\right)$ est vérifiée. Il reste à vérifier l'hypothèse $\left(P_{1}^{\prime}\right)$. En effet, soit $K \in \operatorname{Sym}\left(W_{0}^{1, p}(] 0, \pi[)\right)$ un compact tel que $\theta(K) \geqslant n+1$. Posons $\tilde{K}=\left\{\frac{u}{\|u\|_{1, p}}, u \in K\right\}$. L'application $u \in K \rightarrow \frac{u}{\|u\|_{1, p}} \in \tilde{K}$ est continue impaire, donc $\theta(K) \leqslant \theta(\tilde{K})$, de sorte que $\tilde{K} \in A_{n+1}(\theta)$. D'après (4.2), on a $\min _{u \in \tilde{K}} \int_{0}^{\pi} k(x)|u|^{p} \leqslant \frac{1}{\lambda_{n+1}(\theta, k)}$, puisque $\tilde{K}$ est compact, il existe $u_{0} \in K$ tel que

$\frac{\int_{0}^{\pi} k(x)\left|u_{0}\right|^{p}}{\int_{\left(P_{1}^{\prime}\right) .}^{\pi}\left|u_{0}^{\prime}\right|^{p}} \leqslant \frac{1}{\lambda_{n+1}(\theta, k)}$, donc $u_{0} \in A$, il en résulte que $K \cap A \neq \emptyset$, d'où 
Sur un théorème de point critique et application à un problème de non-résonance

2) et 3) D'après le théorème 5 , la fonctionnelle $\Phi_{p}$ satisfait la condition (PS). Posons

$$
A=\left\{u \in W_{0}^{1, p}(\Omega) ; \lambda_{2}(k) \int_{\Omega} k(x)|u|^{p} \leqslant \int_{\Omega}|\nabla u|^{p}\right\}
$$

et désignons par $\phi_{1}$ la première fonction propre positive associée à $\lambda_{1}(l)$ telle que $\left\|\phi_{1}\right\|_{1, p}=1$. Montrons que : i) $\lim _{|t| \rightarrow+\infty} \Phi_{p}\left(t \phi_{1}\right)=-\infty$.

ii) $\Phi_{p}$ est coercive sur $A$ (c'est à dire $\Phi_{p}(u) \rightarrow+\infty$ lorsque $\|u\|_{1, p} \rightarrow+\infty$ et $u \in A$ ).

En effet : i) D'après $F_{\left(\lambda_{1}, \lambda_{2}\right)}$, on a

$$
\Phi_{p}\left(t \phi_{1}\right) \leqslant \int_{\Omega} \frac{\left|t \nabla \phi_{1}\right|^{p}}{p}-\int_{\Omega} \frac{l-\varepsilon}{p}\left|t \phi_{1}\right|^{p}+\int_{\Omega} d_{\varepsilon}(x)-\left\langle h, t \phi_{1}\right\rangle .
$$

Comme $\int_{\Omega} l(x)\left|\phi_{1}\right|^{p}=\frac{1}{\lambda_{1}(l)}$ et $\int_{\Omega}\left|\phi_{1}\right|^{p} \leqslant \frac{1}{\left.\lambda_{1}(1)\right)}$, on déduit que

$$
\Phi_{p}\left(t \phi_{1}\right) \leqslant \frac{1}{p} \mu|t|^{p}+\int_{\Omega} d_{\varepsilon}(x)-\left\langle h, t \phi_{1}\right\rangle
$$

où $\mu=1-\frac{1}{\lambda_{1}(l)}+\frac{\varepsilon}{\lambda_{1}(1)}$. D'après la proposition 6 , on a $1-\frac{1}{\lambda_{1}(l)}<0$, donc l'affirmation i) est vérifiée pour $\varepsilon$ assez petit tel que $\mu<0$.

ii) D'après $\left(F_{\lambda_{1}, \lambda_{2}}\right)$, pour tout $u \in W_{0}^{1, p}(\Omega)$, on a

$$
\Phi_{p}(u) \geqslant \frac{1}{p}\|u\|_{1, p}^{p}-\frac{1}{p} \int_{\Omega}(k(x)+\varepsilon)|u|^{p}-\int_{\Omega} d_{\varepsilon}(x)-\|u\|_{1, p}\|h\|_{-1, p^{\prime}} .
$$

Si $u \in A$, alors

$$
\Phi_{p}(u) \geqslant \frac{1}{p} \mu^{\prime}\|u\|_{1, p}^{p}-\int_{\Omega} d_{\varepsilon}(x)-\|u\|_{1, p}\|h\|_{-1, p^{\prime}}
$$

où $\mu^{\prime}=\left(1-\frac{1}{\lambda_{2}(k)}-\frac{\varepsilon}{\lambda_{1}(1)}\right)$. Sous les deux conditions 2$)$ et 3$)$ du théorème 6 , la proposition 6 entraîne que $1-\frac{1}{\lambda_{2}(k)}>0$, donc pour $\varepsilon$ assez petit tel que $\mu^{\prime}>0$, l'affirmation ii) est vérifiée.

Finalement, d'après l'affirmation ii) la valeur $\beta:=\inf _{u \in A} \Phi_{p}(u)$ est finie et d'après i) il existe $t_{0}$ assez grand tel que $\alpha:=\max \left\{\Phi_{p}\left(t_{0} \phi_{1}\right), \Phi_{p}\left(-t_{0} \phi_{1}\right)\right\}<$ $\beta$. L'hypothèse $\left(P_{2}\right)$ est donc vérifiée avec $K_{0}=t_{0}\left\{-\phi_{1}, \phi_{1}\right\}$ et $\theta\left(K_{0}\right)=1$. Il reste à vérifier l'hypothèse $\left(P_{1}^{\prime}\right)$. En effet, soit $K \in S y m\left(W_{0}^{1, p}(\Omega)\right)$ un 
compact tel que $\theta(K) \geqslant 2$. Posons $\tilde{K}=\left\{\frac{u}{\|u\|_{1, p}}, u \in K\right\}$, de sorte que $\tilde{K} \in A_{2}(\theta)$. D'après (4.2), on a $\min _{u \in \tilde{K}} \int_{\Omega} k(x)|u|^{p} \leqslant \frac{1}{\lambda_{2}(k)}$. Puisque $\tilde{K}$ est compact, il existe $u_{0} \in K$ tel que $\frac{\int_{\Omega} k(x)\left|u_{0}\right|^{p}}{\int_{\Omega}\left|\nabla u_{0}\right|^{p}} \leqslant \frac{1}{\lambda_{2}(k)}$, par suite $u_{0} \in A$, il en résulte que $K \cap A \neq \emptyset$, d'où $\left(P_{1}^{\prime}\right)$.

4) D'après le théorème 5 , la fonctionnelle $\Phi_{p}$ satisfait la condition (PS). Si $\lambda<\lambda_{1}(\theta, m)$, alors $\Phi_{p}$ est coercive, par suite elle admet un minimum. Si $\lambda_{1}(\theta, m)<\lambda$, alors il existe $n \in \mathbb{N}^{*}$ tel que $\lambda_{n}(\theta, m)<\lambda<\lambda_{n+1}(\theta, m)$. Posons

$$
A=\left\{u \in W_{0}^{1, p}(\Omega) ; \lambda_{n+1}(\theta, m) \int_{\Omega} m(x)|u|^{p} \leqslant \int_{\Omega}|\nabla u|^{p}\right\} .
$$

D'autre part, soit $\varepsilon>0$. Il existe $K^{\prime} \in A_{n}(\theta)$ tel que

$$
\frac{1}{\lambda_{n}(\theta, m)}-\varepsilon \leqslant \min _{u \in K^{\prime}} \int_{\Omega} m(x)|u|^{p} .
$$

Montrons que : i) $\Phi_{p}(u) \rightarrow-\infty$ lorsque $\|u\|_{1, p} \rightarrow+\infty$ et $u \in \mathbb{R} K^{\prime}$. ii) $\Phi_{p}$ est coercive sur $A$.

En effet : Pour tout $u \in W_{0}^{1, p}(\Omega)$, on a

$$
\Phi_{p}(u)=\frac{1}{p}\|u\|_{1, p}^{p}-\frac{\lambda}{p} \int_{\Omega} m(x)|u|^{p}-\langle h, u\rangle .
$$

Si $u \in \mathbb{R} K^{\prime}$, d'après (6.4) on a

$$
\Phi_{p}(u) \leqslant \frac{1}{p}\left(1-\frac{\lambda}{\lambda_{n}(\theta, m)}-\varepsilon \lambda\right)\|u\|_{1, p}^{p}-\langle h, u\rangle .
$$

L'affirmation i) est donc vérifiée pour $\varepsilon$ assez petit. D'autre part, si $u \in A$, alors

$$
\Phi_{p}(u) \geqslant \frac{1}{p}\left(1-\frac{\lambda}{\lambda_{n+1}(\theta, m)}\right)\|u\|_{1, p}^{p}-\langle h, u\rangle
$$

d'où l'affirmation ii). La suite de la démonstration est identique à l'assertion 1) du théorème 6.

5) Cette assertion se démontre de façon identique à l'assertion 1) du théorème 6 . 
Sur un théorème de point critique et application à un problème de non-résonance

\section{Remarques 4.}

1. Grâce à la méthode variationnelle utilisée ici, l'assertion 1 du théorème 6 constitue une amélioration du résultat établi dans [Bo, $\mathrm{Br}, \mathrm{Da}, \mathrm{Ku}]$. De même, les assertions 2 et 3 du théorème 6 sont meilleures que le résultat établi dans [An,Ts2].

2. On retrouve dans l'assertion 4 l'alternative de Fredholm non linéaire par une méthode de minimax et remarquons que les hypothèses $\left(P_{1}\right)$ et $\left(P_{2}^{\prime}\right)$ du corollaire sont vérifiées seulement si $\lambda \neq \lambda_{n}(\theta, m)$ pour tout $n \in \mathbb{N}^{*}$.

3. L'assertion 5) du théorème 6 est établie par Costa et Oliveira dans $[\mathrm{Co}, \mathrm{Ol}]$. Nous retrouvons ce résultat par une autre méthode qui n'utilise pas le caractère linéaire de l'opérateur

\section{Bibliographie}

[Ah,La,Pa] Ahmad (S.), Lazer (A.C.), Paul (J.L.). - Elementary critical point theory and perturbations of elliptic boundary value problems at resonance, Indiana Univ. Math. J. 25 (1976), 933-944.

[An1] ANANE (A.). - Simplicité et isolation de la première valeur propre du p-laplacien avec poids, C.R. Acad. Sci., Paris, t. 305, Série I (1987), 725728.

[An2] ANANE (A.). - Etude des valeurs propres et de la résonance pour l'opérateur p-laplacien, Thèse de Doctorat, Université Libre de Bruxelles, 1987.

[An.Go] ANANE (A.) \& Gossez (J.-P.). - Strongly nonlinear elliptic problem near resonance, a variational approch, comm, P.D.E., 15(B) (1990), 1141-1159.

[An,Ts1] ANANE (A.) and Tsouli (N.). - On the second eigenvalue of the p-laplacian, Pitman Res. Notes in Math., 343 (1996), 1-9.

[An,Ts2] AnANe (A.) and Tsouli (N.). - On a nonresonance condition between the first and the second eigenvalue for the p-laplacian, Accepted in International Journal of Mathematics and Mathematical Sciences, 2000.

[Az] AzızıEH (C.). - Méthodes variationnelles et spectre du p-laplacien, Mémoire de Licence, Univercité Libre de Bruxelles, 1997.

[Bo,Dr,Da,Ku] Boccardo (L.), Drabek (P.), Giachetti (D.) and Kucera (M.). Generalisation of fredholm alternative for nonlinear differential operators, Nonli., 10 (1986), 1083-1103.

[Br,Ni] Brezis (H.) and NiRENBERG (L.). - Characterisations of the ranges of some nonlinear operators and applications to boundary value problems, Ann. Scuola Norm. Sup. Pisa, 5 (1978), 225-326.

[Ca] CAC (N.P.). - On elliptic boundary value problems at double resonance, J. Math. Anal. Appl., 132 (1988), 473-483.

[Ch] Chakrone (O.). - Thèse de Doctorat, Faculté des Sciences, Oujda, Maroc (1998). 
[Co,Ol] Costa (D.G.) and Oliveira (A.). - Existence of solutions for a class of semilinear problems at double resonance, Boll. Soc. Bras. Mat., 19 (1988), 21-37.

[Cu,De,Go] Cuesta (M.), De Figueiredo (D.) and Gossez (J.P.). - The beginning of the Fucik spectrum for the p-Laplacian, J. Diff. Equat., 159 (1999), 212-238.

[De] DE Figueiredo (D.). - Lectures on the Ekeland variational principe with applications and detours, TATA Institute, Springer, 1989.

[De,Go] De Figueiredo (D.) \& Gossez (J.P.). - Strict monotonicity of eigenvalues and unique continuation, Comm. Part. Diff. Equat., 17 (1992), 339-346.

[Dei] Deimling (K.). - Nonlinear Functional Analysis, Springer Verlag, New York-Berlin-Heidelberg, 1985.

[Do] DoLPH (C.L.). - Nonlinear integral equations of the Hammerstein type, Trans. Amer. Math. Soc., 66 (1949), 289-307.

[Go,Lo] Gossez (J.P.) and Loulit (A.). - A note on two notions of unique continuation, Bull. Soc. Math. Belg., 45 (1993), Ser. B., 257-267.

[Go,Mo] Gossez (J.-P.), MoussaouI (M.). - A note on nonresonance between consecutive eigenvalues for a semilinear elliptic problem, Pitman Res. Notes in Math., 343 (1996), 155-166.

[Lo] Loulit (A.). - Inégalités avec poids et problèmes de continuation unique, Thèse de Doctorat, Université Libre de Bruxelles, 1995.

[Mo] Moussaoui (M.).- Questions d'existence dans les problèmes semilinéaires elliptiques, Thèse de Doctorat, Université Libre de Bruxelles, 1991.

[Ot1] OTANI (M.). - A remark on certain nonlinear elliptic equations, Proc. Fac. Sci. Tokai Univ., 19 (1984), 23-28.

[Ot2] OTANI (M.). - On certain second order ordinary differential equations associated with Sobolev-Poincaré-type inequalities, Th. Meth. and Appl. 8 (1984), 1255-1270.

[Pi,Ma] Del Pino (M.A.) and Manasevich (R.F.). - Global Bifurcation from the eigenvalues of the p-laplacian, Journal of Diff. Equat., 92, 226-251 (1991).

[Ra] RABINOwITZ (P.H.). - Minimax methods in critical point theory with applications to differential equations, CBMS Reg. Conf. Series in Math., $\mathrm{n}^{\circ}$ 65, Amer. Math. Soc., Providence, Rhode Island (1986).

[Th] THEws (K.). - Nontrivial solution of elliptic equations at resonance, Proc. R. Soc. Edinb., 85A (1980), 119-129.

[Z] ZELATI (V.C.). - A (short) introduction to critical point theory, ICTP (Trieste), 1997.

[Ze] ZEIDLER (E.). - Nonlinear functional analysis and its applications, Tome III, Variational Methods and Optimization, Springer-Verlag, Berlin (1985). 